\title{
Estrategias para el control \\ del tabaco con perspectiva de género en América Latina
}

Gabriela Regueira, Psic, (I) Nery Suárez-Lugo, Psic, D en C Econ, ${ }^{(2)}$ Silvia Jakimczuk, MC. ${ }^{(3)}$

\author{
Regueira G, Suárez-Lugo N, Jakimczuk S. \\ Estrategias para el control del tabaco \\ con perspectiva de género en América Latina. \\ Salud Publica Mex 2010;52 supl 2:S315-S320.
}

\section{Resumen}

En América Latina, la epidemia del tabaquismo en las mujeres es una realidad ya instalada. Esto se vincula al cambio en el estatus social y económico de las mujeres y el accionar de la industria tabacalera para captar este segmento del mercado. El Convenio Marco para el Control del Tabaco tiene el potencial de ser un tratado de importancia para las mujeres si en la aplicación de sus artículos se incorpora perspectiva de género. Los tratados de derechos de las mujeres también representan una herramienta útil para detener esta epidemia. Es esencial la participación plena y significativa de las mujeres en este proceso.

Palabras clave: tabaquismo; mujeres; Convenio Marco para el Control del Tabaco;América Latina

\author{
Regueira G, Suárez-Lugo N, Jakimczuk S. \\ Tobacco control strategies from \\ a gender perspective in Latin America.
}

Salud Publica Mex 2010;52 suppl 2:S315-S320

\section{Abstract}

In the Latin America region, the tobacco epidemic among women is a fact. This is related to the change in social and economic status among women and the efforts of the tobacco industry to capture their market share. The FCTC has the potential to be an important treaty for women if its articles include a gender perspective. Women's rights treaties, also represent an important tool to stop the epidemic. Women's participation is key in this process.

Key words: smoking; women; Framework Convention on Tobacco Control; Latin America

\section{Las mujeres y la epidemia del tabaquismo}

En el mundo, casi mil millones de varones y cerca de 250 millones de mujeres fuman habitualmente. ${ }^{1}$ Las tendencias mundiales indican que la prevalencia en el consumo de tabaco en varones se encuentra en un lento descenso, mientras que el consumo de tabaco en las mujeres seguirá aumentando, revirtiéndose lo que hasta ahora se consideraba una epidemia masculina. ${ }^{2}$

En el incremento del consumo entre las mujeres a nivel mundial, América Latina no es una excepción, sino una realidad ya instalada. Aunque el consumo de tabaco entre las mujeres varía en los países latinoamericanos, en todos se observa una tendencia en crecimiento.

(I) Coordinación de la Red Latinoamericana y del Caribe de Mujeres para el Control del Tabaco - INWAT LAC. Buenos Aires, Argentina.

(2) Dirección en Sistemas y Servicios de Salud, Escuela Nacional de Salud Pública. La Habana, Cuba.

(3) Comisión Directiva de la Unión Antitabáquica Argentina. Buenos Aires, Argentina.

Fecha de recibido: 26 de marzo de 2010 - Fecha de aceptado: 29 de abril de 2010 Solicitud de sobretiros: Psic. Gabriela Regueira. Juana Azurduy 2157, (8000) Bahía Blanca, Buenos Aires, Argentina. Correo electrónico: inwatlac@live.com.ar 
En Chile, Argentina, Brasil, Uruguay, Cuba y Venezuela se encuentran los mayores niveles de consumo de tabaco entre la población femenina. ${ }^{3}$ Especialmente las jóvenes han superado en consumo de tabaco a los varones, según se señala en la Encuesta Mundial de Tabaquismo en Jóvenes. ${ }^{4}$

Esta situación también incide en las tasas de mortalidad y morbilidad de nuestros países, donde se reduce la expectativa de vida de las mujeres como consecuencia del consumo de tabaco.

Varones y mujeres tienen diferentes problemas de salud no sólo por pertenecer biológicamente a sexos distintos, sino también por vivir en sociedades que interpretan inequitativamente las diferencias entre sexos.

La evidencia científica muestra claramente que el tabaquismo tiene efectos específicos en la salud de las mujeres que las afectan de forma diferente que al varón, y que permiten afirmar no sólo su vinculación con el cáncer, las enfermedades cardiovasculares y pulmonares, sino también consecuencias en la salud reproductiva, en su condición de procreadoras y en los riesgos de la exposición a humo de tabaco ajeno, con el consecuente coste económico que estas enfermedades tienen para las mujeres y sus familias. ${ }^{5}$

El uso y explotación de la mano de obra de las mujeres y las niñas en el cultivo y procesamiento de la hoja de tabaco representan otro aspecto clave de esta problemática de salud pública, pues se enfrentan a condiciones laborales precarias e inseguras, con los riesgos que esto conlleva para su salud. ${ }^{6}$

La desigualdad en salud entre varones y mujeres se origina por la distribución de roles sociales y relaciones de poder que derivan en distintos estilos de vida, prácticas preventivas y de riesgo. Las mujeres son las más perjudicadas, ya que tienen en general una peor situación económica y social, factores que inciden directamente sobre la salud.

Las mujeres en situación de inequidad, pobreza y bajo nivel educativo son el grupo más vulnerable frente a esta epidemia. ${ }^{6}$

Hay una mayor exposición en la adolescencia en situación de desventaja a los factores que incrementan el riesgo de fumar entre las jóvenes. Aquellas que han sufrido violencia familiar se hacen fumadoras con mayor frecuencia que los chicos. ${ }^{7}$

La población en situación de desventaja se beneficia también menos con las medidas de control. La fuerte cultura de fumar, ausencia de líderes con rol modélico, amplio y fácil acceso a los cigarrillos, pocas alternativas de ocio y escaso acceso a servicios de cesación, hacen difícil que las mujeres dejen de fumar.

Las dificultades en acceder a los servicios de salud por carencia y elevado costo, la pobreza, el hambre y la desnutrición, la coexistencia de enfermedades transmisibles con enfermedades no trasmisibles, los escasos derechos laborales y sociales, así como la alta frecuencia de mujeres amas de casa y dependientes de la economía informal, agravan el costo de la epidemia tabáquica en lo personal, familiar y social en la región, por lo que constituye sin lugar a dudas uno de los más importantes problemas de salud pública. ${ }^{8}$

\section{La industria del tabaco y su seducción a la mujer}

Las mujeres han ido transformando su imagen y su rol en la sociedad desde la adquisición de derechos civiles fundamentales, como la posibilidad de votar, hasta la actualidad, donde la mujer tiene su propio espacio. Factores como la urbanización, estilos de vida urbanos, participación en la población económicamente activa y el mayor acceso a la educación y a los medios de comunicación traen como resultado un incremento en la exposición a la publicidad.

Con el advenimiento de esta nueva mujer en los últimos años también se ha producido el establecimiento de factores favorecedores del tabaquismo en las mujeres. Esto ha brindado nuevas posibilidades a la industria tabacalera para explotar diferentes estrategias de mercadotecnia focalizadas en este creciente segmento del mercado. ${ }^{9}$

Debido a que en los países desarrollados comienzan a disminuir las ventas a causa del abandono del tabaco por parte de los varones y de los que empiezan a enfermar y morir como consecuencia de enfermedades vinculadas a dicho producto, la industria ha tenido que apuntar al mercado femenino, especialmente en los países en vías de desarrollo. ${ }^{10}$

Los documentos "secretos" de la industria del tabaco detallan extensos planes de mercadotecnia y publicidad para promover sus productos a las mujeres alrededor del mundo. ${ }^{11}$ De acuerdo con éstos, la importancia asignada a la mujer se debe a factores como el hecho de que son pocas las que dejan de fumar una vez que se inician en el consumo, a que cada vez es mayor el número de ellas que comienzan a fumar y a que entre las jóvenes se cuenta con una creciente proporción de mujeres que fuman. ${ }^{12}$

La industria del tabaco investiga, utiliza y explota las cuestiones relacionadas con el género y sus cambios para promover y vender el tabaco. Conoce que los motivos por los cuales fuman las mujeres son diferentes a los motivos por los cuales fuman los varones. Para las mujeres tienen especial importancia la percepción de la imagen corporal y el control del peso, neutralizar sentimientos negativos como el estrés y la ansiedad, así 
como la autoestima. En las mujeres también es diferente el proceso de iniciación y mantenimiento, y es más difícil el abandono del tabaquismo. ${ }^{13}$

Apoyada en esta evidencia, la industria desarrolla e implementa estrategias de comercialización para lograr la feminización del consumo de tabaco e incrementar de esta manera sus ventas y ganancias. ${ }^{14}$

La mujer se convirtió en la garantía para la rentabilidad de la industria del tabaco y la publicidad se encargó de reclutar más mujeres para su consumo, pues son éstas el grupo diana fundamental al que dirige sus campañas publicitarias en particular y sus estrategias de mercadotecnia en general, y se transforma al cigarrillo y al acto de fumar en un símbolo social que seduce a las mujeres, centrado fundamentalmente en los aspectos siguientes: relación de fumar con la libertad femenina, emancipación y empoderamiento; amplia variedad de imágenes que asocian los cigarrillos con estereotipos de ser mujer, como famosas cantantes y actrices que aparecen fumando su marca de cigarrillos, ya sea en el escenario o en la pantalla; asociación del acto de fumar con sensualidad, esbeltez, sofisticación y atractivo sexual; con autonomía, modernidad, ser "una mujer real" o "una mujer moderna" e, incluso, tener relaciones lésbicas, por citar las más importantes. ${ }^{15}$

La industria tabacalera está implementando en América Latina las mismas estrategias de mercadotecnia dirigidas a la mujer que ha estado utilizado durante años en los países desarrollados, adaptándolas al mercado regional. En todos los países del mundo esta industria ha ido modificando las estrategias desde el momento que comenzó a dirigirse a las mujeres hasta la actualidad, al desarrollar un amplio rango de mensajes y productos para llegar a los diferentes segmentos del mercado femenino. ${ }^{16}$

La industria tabacalera sabe que hay desigualdades entre las mujeres jóvenes y apunta a cada grupo de ellas en función de su mayor vulnerabilidad, pero de manera general maneja algunas claves para atraer al tabaquismo a la mujer adolescente y joven, con nociones como las de que fumar es un símbolo de independencia, libertad, emancipación, ayuda a conseguir la ansiada delgadez y a relacionarse, a "ligar", es decir, a tener mayor atractivo sexual, promete felicidad, alegría, buena forma física y poder, es equiparable a aventura, inconformismo y ayuda a hacer frente a los problemas y, por supuesto, a controlar el estrés. ${ }^{17}$

La moda ha sido, por tanto, una herramienta útil para incrementar el mercado de mujeres consumidoras de tabaco, de ahí que las compañías tabacaleras asocien los productos de tabaco a la imagen de supermodelos que además son muy delgadas, haciendo que en ese rol modélico se refuerce la idea de que fuman para perma- necer delgadas y, por lo tanto, las jóvenes quieran ser como ellas. ${ }^{18}$

La publicidad y promoción que realizan las compañías tabacaleras buscan fomentar el consumo de tabaco entre quienes se inician o desean cambiar de marca, incitar a quienes consumen tabaco a fumar más y reducir su motivación para abandonar el tabaquismo. ${ }^{19}$

Un ejemplo evidente de la información engañosa de la industria tabacalera en esa dirección es el uso de términos como "light" o "slim", que asocian los productos de tabaco con dos aspiraciones femeninas clave como ser delgada y ser atractiva. Las mujeres tradicionalmente han sido incluidas en el segmento light. ${ }^{20}$

El empleo de la publicidad en actividades y revistas femeninas se unió a la presencia de marcas con nombre de mujer, dentro de una atractiva estrategia.

La marca Virginia Slims ha sido diseñada especialmente para el mercado femenino. Los documentos de la industria tabacalera señalan que en América Latina el grupo diana de la marca es la mujer adulta joven que busca un símbolo femenino. En estos documentos también se indican las razones que justifican su lanzamiento en la región, destacándose la importancia de la imagen y la belleza en las mujeres latinoamericanas, y por el hecho de que parecen sentirse cómodas con la idea de feminidad y generalmente siguen la moda. ${ }^{21}$

La industria del tabaco se vale de todas estas estrategias para seducir a la mujer, y el estudio cuidadoso de éstas es la clave para poder ser eficaces en la reducción del tabaquismo en la población femenina.

\section{Género y Convenio Marco para el Control del Tabaco}

El Convenio Marco para el Control del Tabaco (CMCT) ${ }^{22}$ representa una herramienta fundamental en el campo de la salud pública para reducir el tabaquismo y empoderar a las mujeres, ya que reconoce la importancia de incorporar un enfoque de género en la programación, establecimiento de políticas y realización de estudios sobre el tabaco en apoyo al avance legislativo.

En su preámbulo menciona de manera explícita a las mujeres y expresa su preocupación por el incremento del consumo de tabaco entre mujeres y niñas en todo el mundo, y reclama la necesidad de estrategias de control del tabaco específicas de género. Asimismo refuerza la necesidad de que las mujeres participen en la formulación y aplicación de políticas de control del tabaco, y destaca la contribución especial que hacen las organizaciones no gubernamentales a los esfuerzos nacionales e internacionales en ese empeño. Hace explícita mención a las asociaciones de mujeres, entre otras organizaciones de la sociedad civil. 
El CMCT tiene todo el potencial para ser un importante tratado que proteja la salud de las mujeres, particularmente si en la aplicación de sus artículos se incluye la perspectiva de género. ${ }^{23}$ En el Artículo 4 se menciona la necesidad de adoptar medidas para que, cuando se elaboren estrategias de control del tabaco, se tengan en cuenta los riesgos relacionados específicamente con el género.

El CMCT representa también una herramienta fundamental para los gobiernos y la sociedad civil en su lucha por frenar la epidemia del tabaquismo. La sociedad civil puede contribuir especialmente al lograr involucrar a los tomadores de decisiones y líderes de opinión, al educar al público y a las personas acerca de sus derechos y cómo ejercerlos, y al formar redes de organizaciones que puedan facilitar el uso de los instrumentos legislativos existentes. ${ }^{24}$

En su preámbulo, el CMCT incorpora disposiciones de la Convención para la Eliminación de Toda Forma de Discriminación Contra la Mujer (CEDAW), que fue adoptada en 1979 por la Asamblea General de Naciones Unidas, y que constituye una herramienta internacional para los derechos de las mujeres. ${ }^{25}$

En sus artículos, la CEDAW urge a que se tomen todas las medidas oportunas para eliminar la discriminación contra la mujer en el ámbito de la atención sanitaria. Además, manifiesta que en todas las políticas y programas que afecten a la salud de las mujeres debe integrarse una perspectiva de género y señala que las mujeres deben estar implicadas en la planificación, implantación y evaluación de esas políticas y programas; les da el derecho a participar en la vida pública y en la toma de decisiones y les asegura condiciones de trabajo seguras. ${ }^{26}$

Aunque el tabaco no se menciona específicamente en la Convención, ésta representa una herramienta de derechos humanos que, utilizada en conjunto con el $\mathrm{CMCT}$, permite potenciar sus efecto ${ }^{27}$ para proteger la salud de las mujeres frente a la epidemia devastadora del tabaquismo, desarrollando una política firme y eficaz basada en el binomio educación-legislación con énfasis en la prevención como medida de mayor impacto potencial para reducir la morbilidad y mortalidad.

\section{América Latina, tabaco y género}

Los países de América Latina enfrentan grandes retos para abordar la epidemia del tabaquismo desde una perspectiva de género. Si bien en los últimos años se han obtenido importantes avances en la región vinculados a este tema, todavía no se ha logrado incluir de manera plena en la agenda del control del tabaco y menos aún en la agenda de las organizaciones de salud de las mujeres.

Tal como lo demuestra el análisis de los documentos internos de la industria tabacalera, ésta se ha movido con gran rapidez para comprender los aspectos de la comercialización del tabaco desde una perspectiva de género, a diferencia de las investigaciones, programas y políticas de control del tabaco, que lentamente han iniciado su camino a la incorporación de enfoques basados en el género.

La situación social, económica y cultural de las mujeres varía significativamente de un país a otro, al igual que el consumo de tabaco de mujeres y jóvenes. La comprensión de estos puntos es fundamental para prevenir y/o reducir el consumo de tabaco entre las mujeres y las jóvenes.

Es primordial también promover la recopilación de datos adecuados sensibles al sexo y al género y la adaptación de los programas y las políticas en la región de manera que lleguen a los grupos de mujeres más vulnerables. Asimismo, se debe promover el empoderamiento de estas mujeres.

En América Latina, la promoción e implementación de ambientes libres de humo de tabaco es la medida más exitosa en los últimos años. Pero también se requiere la adopción de otras políticas del CMCT que a nivel mundial han demostrado tener impacto en la prevención y reducción del consumo de tabaco. Estas incluyen, por ejemplo, la prohibición de la publicidad, el aumento de los precios de los productos de tabaco, y la regulación de la venta, entre otras, medidas que luego deben ser reforzadas con campañas de educación y comunicación.

Las organizaciones de salud de la mujer representan un importante sector de la sociedad civil que se debe involucrar en el desarrollo de programas y políticas de control de tabaco por su experiencia y el importante rol que cumplen en la promoción de la salud de las mujeres y su empoderamiento político, lo que facilitaría la incorporación de una perspectiva de género. ${ }^{28}$ Esto se puede lograr a través de la concientización y movilización de las mujeres activistas y del movimiento de la salud. ${ }^{29}$

Un ejemplo de trabajo con perspectiva de género aplicado al control del tabaco, desde la sociedad civil, está dado por la International Network of Women Against Tobacco, (INWAT), Red Internacional de Mujeres frente al Tabaco.

INWAT es una red global de especialistas en control de tabaco dedicadas a promover la salud y equidad entre 
las mujeres y jóvenes de todo el mundo, por vía de la eliminación del uso del tabaco y de la exposición pasiva al humo de tabaco ajeno. Fue creada en el año 1990 por mujeres líderes en el control del tabaco, durante la 7th. Conferencia Mundial Tabaco o Salud en Perth (Australia). Desde su inicio, la mujer latinoamericana tuvo representación. La Red surge ante la preocupación de estas mujeres frente a la falta de intervenciones, investigación e intercambios de conocimiento y experiencias en el control del tabaco que fueran sensibles al género.

INWAT, a través de sus acciones alrededor del mundo, ha situado en el centro de todos los esfuerzos de control del tabaco, el empoderamiento y el liderazgo de las mujeres, que son esenciales para el éxito de los programas nacionales y del CMCT. Actualmente cuenta con más de 1800 miembros en 100 países y actúa proporcionando contactos individuales e institucionales, principalmente de mujeres especializadas en control del tabaco, recogiendo y distribuyendo información a nivel mundial.

Además comparte estrategias para contrarrestar la promoción y la publicidad del tabaco; apoya que los programas específicos de prevención, deshabituación y control del tabaco se desarrollen con enfoque de género; asesora en la organización y planificación de conferencias sobre control del tabaco; colabora en el desarrollo de publicaciones, y promueve el liderazgo de las mujeres en ese campo.

Los principales logros de INWAT han estado vinculados a dar impulso a la agenda de investigación y formación sobre mujeres y tabaco; promover la inserción de la perspectiva de género en la agenda internacional sobre el tema; demostrar la utilidad del análisis de género y estimular la incorporación de esta perspectiva tanto en publicaciones como en programas y políticas de control de tabaco.

Ha contribuido en la redacción del CMCT, asegurándose que incluya medidas beneficiosas para las mujeres y permita involucrarlas en el proceso de toma de decisiones que pueden afectarlas. También ha prestado especial interés en apoyar el proceso de negociación, ratificación e implementación.

Durante la $13^{\circ}$ Conferencia Mundial Tabaco o Salud que se realizó en la ciudad de Washington, EU, INWAT acordó brindar su apoyo para el desarrollo de redes regionales. Siguiendo esta iniciativa, el 7 de septiembre de 2007 en Río de Janeiro, Brasil, durante el Primer Congreso Latinoamericano de la SRNT \& Segunda Conferencia Iberoamericana sobre Control del Tabaco, se creó la Red Latinoamericana y del Caribe de Mujeres para el Control del Tabaco (INWAT-Latinoamérica y del Caribe) que realizó su primera reunión en Mumbai, India, en el transcurso de la $14^{\circ}$ Conferencia Mundial Tabaco o Salud.

Actualmente cuenta con más de 100 miembros en 16 países de la región, y se perfila como un movimiento activo que va ganando fuerza cada día.

\section{Conclusiones}

En América Latina, la epidemia del consumo de tabaco en las mujeres exige grandes retos a largo plazo para el control del tabaco.

La industria sigue avanzando y se dirige de manera cada vez más agresiva a las mujeres y las niñas para incrementar su rentabilidad en la región.

El CMCT ha demostrado ser una herramienta de suma importancia para frenar la epidemia en las mujeres. Si bien todavía resta mucho por conocer acerca del consumo de tabaco en relación con el género, expresa su inquietud al respecto y promueve medidas para el control del tabaco sensibles al género al establecer las bases de acción.

Es fundamental que, en el corto plazo, se realicen estudios regionales que incluyan perspectiva de género. La realidad exige actuar ahora, pues así se podrá salvar a millones de niñas y mujeres de morir a causa de enfermedades vinculadas directamente con el consumo de tabaco.

Mientras las evidencias avanzan, hay que continuar utilizando la información disponible para avanzar con sensibilidad en cuestiones de género, propiciando la participación de los movimientos de derechos humanos y de salud de las mujeres en los programas de control de tabaco.

Hay que crear conciencia sobre los efectos del tabaquismo en la salud de la mujer y contrarrestar las campañas publicitarias que tienen como objetivo al público femenino, así como contactar autoridades gubernamentales y parlamentarias para promover leyes, normas y regulaciones que protejan a mujeres y niñas de las consecuencias del tabaquismo.

El empoderamiento y el liderazgo de las mujeres deben situarse en el centro de todos los esfuerzos de control del tabaco y son esenciales para el éxito de los programas nacionales y del Convenio Marco para el Control del Tabaco

\section{Declaración de conflicto de intereses}

Declaramos no tener conflicto de intereses. 


\section{Agradecimientos}

Agradecemos a la Dra. Paola Morello, médica especialista en Pediatría y máster en Salud Pública, y a Dolores Marín, psicóloga especialista en Psicología Clínica, los comentarios críticos al documento.

\section{Referencias}

I. Guindon G, Boisclair E, Boisclair D. Past, current and future trends in tobacco use. [artículo en Internet] March 2003 (2nd ed.) [consultado 2010 enero 20]. Disponible en: http://wwwl.worldbank.org/tobacco/ publications.asp

2. Brundtland GH. Prólogo. Samet JM Samet, Yoon SY (Eds.). Mujeres y la epidemia del tabaco - Retos para el s. XXI. Ginebra: Organización Mundial de la Salud, 200I.

3. Mackay J, Eriksen M, Shafey O, Ross H. The tobacco atlas. 3th ed.Atlanta, Georgia USA:American Cancer Society, World Lung Foundation, 2009. 4. Organización Mundial de la Salud. Encuesta mundial sobre el tabaco en jóvenes [sitio en Internet]. [consultado 2010 enero 30].

Disponible en: http://www.edc.gov/spanish/tabaco.htm-32k

5. United States Surgeon General.Women and smoking: a report of the Surgeon General. Rockville, MD, United States Department of Health and Human Services, Office of the Surgeon General, $200 \mathrm{I}$.

6. Greaves L, Jategaonkar N, Sanchez S. (Eds.) Turning a new leaf: Women, tobacco, and the future. British Columbia Centre of Excellence for Women's Health (BCCEWH) and International Network of Women Against Tobacco (INWAT).Vancouver: British Columbia Centre of Excellence for Women's Health, 2006.

7. Suárez LN, Nahuat R. Comportamiento del tabaquismo en adolescentes de la Escuela Secundaria Técnica No. 10. Cárdenas, Tabasco. 2005. Revista Horizontes Sanitarios 2006; 5 (2): 26-43.

8. Suárez LN. Tabaco o salud: una decisión social. Revista Horizontes Sanitarios 2004; 3(2): |2-2|

9. Gómez A. Debemos enfrentar la epidemia del tabaquismo en las mujeres. Revista Mujer Salud. Red de Salud de las Mujeres Latinoamericanas y del Caribe 2008; 4: 4-II.

10. Bianco E. Mujeres, industria tabacalera y marketing. Revista Mujer Salud. Red de Salud de las Mujeres Latinoamericanas y del Caribe 2004; I:3-7. II. Campaign for Tobacco Free Kids - Action on Smoking and Health. Confie en nosotros. Somos la industria tabacalera. [documento en internet] [consultado 2010 enero 28] Disponible en:http://www. tobaccofreekids.org/campaign/global/framework/docs/TrustUsSpanish.pdf 12. Friedman V.The female smoker market. Lorillard collection. June 28 , 1973. Bates No. $03375503 / 55$ I0. [documento en internet] [consultado 2009 julio 10] Disponible en: http://legacy.library.ucsf.edu/tid/lce9 /e00. 13. Tauras JA, O'Malley PM, Johnston LD. Effects of price and access laws on teenage smoking initiation: A national longitudinal analysis. [artículo en internet] [consultado 2010 enero I2] Disponible en: http://www. impacteen.org/.

14. Organización Panamericana de la Salud. La rentabilidad a costa de la gente. Washington DC: Organización Panamericana de la Salud, 2002.

15. Suárez LN. Enfoque social de la mercadotecnia sanitaria. La Habana: Editorial Ciencias Médicas, 2007: 224-244.

16. Amos A, Haglund M. From social taboo to "torch of freedom": The marketing of cigarettes to women. Tobacco Control 2000; 9(I):3-8.

17. Suárez LN. América Latina: los jóvenes y el tabaquismo. Promotion Education Journal of Health 1995; 2(I): 52-54.

18. World Health Organization. Tobacco free film, tobacco free fashion: Action! World No Tobacco Day. Ginebra:World Health Organization, 2003. 19. Fundación Educación Popular en Salud. Detrás del humo: Una mirada a la industria tabacalera en Chile, sus redes y los límites de la regulación y la transparencia frente a las Elecciones Presidenciales 2009 [informe en internet] [consultado 2010 enero 30] Disponible en: http://www.epes.cl 20. American Heart Association, American Cancer Society Cancer Action Network,American Lung Association, Robert Wood Johnson Foundation, Campaign for Tobacco-Free Kids. Deadly in pink. Big tobacco steps up its targeting of women and girls. [documento en internet] [consultado 2010 febrero 04]. Disponible en: www.tobaccofreekids.org/deadlyinpink 21. Leo Burnett Agency.Virginia Slims: opportunities in Latin America. Philip Morris. June 2, 1994. Bates No. 2500055432/555I. [documento en internet] [consultado 2010 enero 05] Disponible en: http://legacy.library. ucsf.edu/tid/mjy8Ifo0.

22. Organización Mundial de la Salud. Convenio Marco para el Control del Tabaco. Ginebra: Organización Mundial de la Salud, 2003.

23. World Health Organization. Gender and tobacco control:a policy brief. Geneva:World Health Organización, 2007.

24. ErnsterV, Kaufman N, Nichter M, Samet J,Yoon SY. Las mujeres y el tabaco: de la política a la acción. Bol Organ Mundial Salud 200 I;78: 89I-90I. 25. Joossens $L$. From public health to international law: possible protocols for inclusion in the Framework Convention on Tobacco Control. Bulletin of the World Health Organization. Geneva, 2000 [artículo en internet] [consultado 2010 enero 15] Disponible en: http//www.whqlibdoc.who.int/ bulletin/2000/Number

26. Organización de las Naciones Unidas. Convención sobre la Eliminación de todas las formas de Discriminación contra la Mujer (CEDAW) Nueva York, 1979 [documento en internet] [consultado 2010 febrero 04] Disponible en: http://www.un.org/womenwatch/daw/cedaw/text/ sconvention.htm

27. World Health Organization. Sifting the evidence: gender and tobacco control. Geneva:World Health Organization, 2007.

28. Yoon SY.A framework for human rights, gender and tobacco. The Net [serie en internet] Fall $2004 \mathrm{I}-5$ [consultado 2010 febrero 04]. Disponible en: http://www.inwat.org/images/pdfs/NET_2004_nr2.pdf

29. Bianco M. Políticas y herramientas para la movilización en América Latina y el Caribe [documento en internet] [consultado 2010 enero 29]. Disponible en: http://www.feim.org.ar/pdf/publicaciones/Ponencia_Tabaco_ Kobe.pdf 